\title{
Logarithmic inequalities for Fourier multipliers
}

\author{
Adam Osȩkowski
}

Received: 2 September 2011 / Accepted: 9 January 2012 / Published online: 3 November 2012

(C) The Author(s) 2012. This article is published with open access at Springerlink.com

Abstract In the paper we study LlogL estimates for Fourier multipliers resulting from modulation of the jumps of Lévy processes. We exhibit a class of functions $m: \mathbb{R}^{d} \rightarrow \mathbb{C}$, for which the corresponding multipliers $T_{m}$ satisfy the following estimate: for $K>1$, any locally integrable function $f$ on $\mathbb{R}^{d}$ and any Borel subset $A$ of $\mathbb{R}^{d}$,

$$
\int_{A}\left|T_{m} f(x)\right| \mathrm{d} x \leq K \int_{\mathbb{R} d} \Psi(|f(x)|) \mathrm{d} x+\frac{|A|}{2(K-1)},
$$

where $\Psi(t)=(t+1) \log (t+1)-t$. We also present related lower bounds which arise from considering appropriate examples for the Beurling-Ahlfors operator.

Keywords Fourier multiplier $\cdot$ Singular integral $\cdot$ Martingale $\cdot$ Differential subordination

Mathematics Subject Classification (1991) Primary 42B15 - 60G44; Secondary 42B20

\section{Introduction}

The martingale theory is a fundamental tool in obtaining $L^{p}$ bounds for many important singular integrals and Fourier multipliers. The purpose of this paper is to extend this connection to the case of appropriately localized $L \log L$ inequalities. This will be accomplished by the use of a certain duality approach and a related sharp inequality for differentially subordinated martingales.

Partially supported by Polish Ministry of Science and Higher Education (MNiSW) grant IP2011 039571 'Iuventus Plus'.

A. Osȩkowski ( $\varangle)$

Department of Mathematics, Informatics and Mechanics, University of Warsaw,

Banacha 2, 02-097 Warsaw, Poland

e-mail: ados@mimuw.edu.pl 
Recall that for any bounded function $m: \mathbb{R}^{d} \rightarrow \mathbb{C}$, there is a unique bounded linear operator $T_{m}$ on $L^{2}\left(\mathbb{R}^{d}\right)$, called the Fourier multiplier with the symbol $m$, which is given by $\widehat{T_{m} f}=m \hat{f}$. The norm of $T_{m}$ on $L^{2}\left(\mathbb{R}^{d}\right)$ is equal to $\|m\|_{L^{\infty}\left(\mathbb{R}^{d}\right)}$ and it has been long of interest to study those $m$, for which the corresponding Fourier multiplier extends to a bounded linear operator on $L^{p}\left(\mathbb{R}^{d}\right), 1<p<\infty$. In this paper we shall consider the following class of symbols, studied by Bañuelos and Bogdan [1] and Bañuelos et al. [2]. Let $v$ be a Lévy measure on $\mathbb{R}^{d}$ : that is, a nonnegative Borel measure on $\mathbb{R}^{d}$ such that $v(\{0\})=0$ and

$$
\int_{\mathbb{R}^{d}} \min \left\{|x|^{2}, 1\right\} v(\mathrm{~d} x)<\infty
$$

Assume further that $\mu$ is a finite Borel measure on the unit sphere $\mathbb{S}$ of $\mathbb{R}^{d}$ and fix two Borel functions $\phi$ on $\mathbb{R}^{d}$ and $\psi$ on $\mathbb{S}$ which take values in the unit ball of $\mathbb{C}$. We define the associated multiplier $m=m_{\phi, \psi, \mu, \nu}$ on $\mathbb{R}^{d}$ by

$$
m(\xi)=\frac{\frac{1}{2} \int_{\mathbb{S}}\langle\xi, \theta\rangle^{2} \psi(\theta) \mu(\mathrm{d} \theta)+\int_{\mathbb{R}^{d}}[1-\cos \langle\xi, x\rangle] \phi(x) v(\mathrm{~d} x)}{\frac{1}{2} \int_{\mathbb{S}}\langle\xi, \theta\rangle^{2} \mu(\mathrm{d} \theta)+\int_{\mathbb{R}^{d}}[1-\cos \langle\xi, x\rangle] \nu(\mathrm{d} x)}
$$

if the denominator is not 0 , and $m(\xi)=0$ otherwise. Here $\langle\cdot, \cdot\rangle$ stands for the scalar product in $\mathbb{R}^{d}$. As proved in [1] and [2], the Fourier multipliers corresponding to these symbols can be given a martingale representation by the use of transformations of jumps of Lévy processes. Combining this representation with Burkholder's moment inequality (see Theorem 2.1 below), Bañuelos et al. [2] established the following $L^{p}$ estimate. See also Bañuelos and Osȩkowski [5] for related lower bounds.

Theorem 1.1 Let $1<p<\infty$ and let $m=m_{\phi, \psi, \mu, v}$ be given by (1.1). Then for any $f \in L^{p}\left(\mathbb{R}^{d}\right)$ we have

$$
\left\|T_{m} f\right\|_{L^{p}\left(\mathbb{R}^{d}\right)} \leq\left(p^{*}-1\right)\|f\|_{L^{p}\left(\mathbb{R}^{d}\right)} .
$$

The constant $p^{*}-1$ is the best possible.

In particular, this theorem yields sharp bounds for the real and imaginary parts of the Beurling-Ahlfors transform (see Sect. 4).

There is a natural question whether the interplay between the martingale theory and Fourier multipliers can be carried over to other types of estimates. The objective of this paper is to give the affirmative answer to this question. We shall consider the class of LlogL inequalities, which arise naturally when one wants to study $L^{1}$ version of (1.2). Throughout the paper, the functions $\Phi, \Psi:[0, \infty) \rightarrow[0, \infty)$ are given by the formulas

$$
\Phi(x)=e^{x}-1-x \quad \text { and } \quad \Psi(x)=(x+1) \log (x+1)-x .
$$

Define the corresponding $\operatorname{Llog} \mathrm{L}$ class by

$$
\operatorname{LlogL}\left(\mathbb{R}^{d}\right)=\left\{f: \mathbb{R}^{d} \rightarrow \mathbb{C}: \int_{\mathbb{R}^{d}} \Psi(|f(x)|) \mathrm{d} x<\infty\right\} .
$$

The main result of the paper can be stated as follows. 
Theorem 1.2 Fix $K>1$ and let $m=m_{\phi, \psi, \mu, v}$ be given by (1.1), with $\mu, v, \phi$ and $\psi$ satisfying the above assumptions. Then for any $K>1$, any $f \in \operatorname{Llog} L\left(\mathbb{R}^{d}\right)$ and any Borel subset $A$ of $\mathbb{R}^{d}$ we have

$$
\int_{A}\left|T_{m} f(x)\right| d x \leq K \int_{\mathbb{R}^{d}} \Psi(|f(x)|) d x+\frac{|A|}{2(K-1)} .
$$

The reader may wonder why we have used the function $\Psi$ instead of the usual $x \mapsto$ $x \log ^{+} x$ or $x \mapsto x \log x$. Another question which comes into one's mind is whether we can replace the integral on the right by the "local" integral over $A$. We shall see in Sect. 4 that in general the inequality breaks down if we implement any of these two changes. Thus (1.3) is the right form for investigation.

By standard optimization over $K$, Theorem 1.2 leads to the following statement.

Corollary 1.3 Under the assumptions of the above theorem, we have

$$
\int_{A}\left|T_{m} f(x)\right| d x \leq \int_{\mathbb{R}^{d}} \Psi(|f(x)|) d x+\sqrt{2}\left(|A| \int_{\mathbb{R}^{d}} \Psi(|f(x)|) d x\right)^{1 / 2} .
$$

What can be said about the tightness of (1.3) and (1.4)? We strongly believe that these estimates are sharp [(i.e., the constants $(2(K-1))^{-1}$ in (1.3) and $\sqrt{2}$ in (1.4) cannot be decreased]. Unfortunately, we have not been able to prove this. Nevertheless, we shall show the sharpness of the martingale inequality on which the argumentation is based. We shall also present examples for the Beurling-Ahlfors transform, which will yield the following statement.

Theorem 1.4 For any $K>2 / \pi$ there is a multiplier $m: \mathbb{C} \rightarrow \mathbb{R}$ from the class (1.1), a Borel subset $A$ of $\mathbb{C}$ and a function $f \in \operatorname{Llog} L(\mathbb{C})$ for which

$$
\int_{A}\left|T_{m} f(z)\right| d z=K \int_{\mathbb{C}} \Psi(|f(z)|) d z+\frac{|A|}{\pi(K \pi-2)} .
$$

Furthermore, for any $c<2 / \pi$ there is a multiplier $m: \mathbb{C} \rightarrow \mathbb{R}$ from the class (1.1), a Borel subset $A$ of $\mathbb{C}$ and a function $f \in \operatorname{Llog} L(\mathbb{C})$ for which

$$
\int_{A}\left|T_{m} f(z)\right| d z>\int_{\mathbb{C}} \Psi(|f(z)|) d z+c\left(|A| \int_{\mathbb{C}} \Psi(|f(z)|) d z\right)^{1 / 2} .
$$

A few words about the proof and the organization of the paper. The heart of the matter lies in proving a certain martingale inequality which can be regarded as a dual to (1.3) and is of independent interest. This is done in the next section. Section 3 is devoted to the proof of (1.3). We also discuss there the possibility of extending this estimate to the vector valued setting. In the final part of the paper we study the lower bound for the constants in (1.3) and (1.4).

\section{A martingale inequality}

As mentioned in the Sect. 1, the results of this paper depend heavily on a certain inequality for differentially subordinated martingales. Let us start with introducing the necessary 
probabilistic background and notation. Assume that $(\Omega, \mathcal{F}, \mathbb{P})$ is a complete probability space, equipped with $\left(\mathcal{F}_{t}\right)_{t \geq 0}$, a nondecreasing family of sub- $\sigma$-fields of $\mathcal{F}$, such that $\mathcal{F}_{0}$ contains all the events of probability 0 . Let $X, Y$ be two adapted martingales taking values in a certain separable Hilbert space $(\mathcal{H},|\cdot|)$, which may and will be taken to be equal to $\ell_{2}$. As usual, we assume that the processes have right-continuous trajectories that have limits from the left. The symbol $[X, Y]$ will stand for the quadratic covariance process of $X$ and $Y$. See e.g. Dellacherie and Meyer [9] for details in the case when the processes are real-valued, and extend the definition to the vector setting by $[X, Y]=\sum_{k=0}^{\infty}\left[X^{k}, Y^{k}\right]$, where $X^{k}, Y^{k}$ are the $k$-th coordinates of $X, Y$. Following Bañuelos and Wang [6] and Wang [17], we say that $Y$ is differentially subordinate to $X$, if the process $\left([X, X]_{t}-[Y, Y]_{t}\right)_{t \geq 0}$ is nonnegative and nondecreasing as a function of $t$.

A celebrated theorem of Burkholder [7] compares the $L^{p}$-norms of differentially subordinated martingales. We would like to mention that the result was originally formulated in the discrete-time case, and the extension below is due to Wang [17] (see also [8]). We use the notation $\|X\|_{p}=\sup _{t \geq 0}\left\|X_{t}\right\|_{p}$ for $1 \leq p \leq \infty$.

Theorem 2.1 Assume that $X, Y$ are $\mathcal{H}$-valued martingales such that $Y$ is differentially subordinate to $X$. Then for $1<p<\infty$ we have

$$
\|Y\|_{p} \leq\left(p^{*}-1\right)\|X\|_{p},
$$

where $p^{*}=\max \{p, p /(p-1)\}$. The constant $p^{*}-1$ is the best possible.

In the limit case $p=1$ the moment inequality does not hold any more, but we have the following substitute, established by the author in [14] in the discrete-time case; see [15] and [16] for the continuous time version.

Theorem 2.2 Assume that $X, Y$ are $\mathcal{H}$-valued martingales such that $Y$ is differentially subordinate to $X$. Then for any $K>1$,

$$
\|Y\|_{1} \leq K \sup _{t \geq 0} \mathbb{E}\left|X_{t}\right| \log \left|X_{t}\right|+L(K),
$$

where

$$
L(K)= \begin{cases}\frac{K^{2}}{2(K-1)} \exp \left(-K^{-1}\right) & \text { if } K \leq 2 \\ K \exp \left(K^{-1}-1\right) & \text { if } K>2\end{cases}
$$

The constant is the best possible.

We shall study a certain version of this result; in fact, to obtain the localized estimate (1.3), we need to consider the following dual bound.

Theorem 2.3 Assume that $X, Y$ are $\mathcal{H}$-valued martingales such that $\|X\|_{\infty} \leq 1$ and $Y$ is differentially subordinate to $X$. Then for any $K>1$ we have

$$
\sup _{t \geq 0} \mathbb{E} \Phi\left(\left|Y_{t}\right|\right) \leq \frac{1}{2 K(K-1)}\|X\|_{1} .
$$

The constant $1 /(2 K(K-1))$ is the best possible.

The proof rests on Burkholder's method: we shall deduce the inequality (2.2) from the existence of a family $\left\{U_{K}\right\}_{K \in(1, \infty)}$ of certain special functions given on the set 
$S=\{(x, y) \in \mathcal{H} \times \mathcal{H}:|x| \leq 1\}$. To this end, we first study an auxiliary object: a function $u_{\infty}: \mathcal{H} \times \mathcal{H} \rightarrow \mathbb{R}$ given by

$$
u_{\infty}(x, y)= \begin{cases}0 & \text { if }|x|+|y|<1 \\ (|y|-1)^{2}-|x|^{2} & \text { if }|x|+|y| \geq 1\end{cases}
$$

We shall need the following properties of this function.

Lemma 2.4 (i) There is an absolute constant $A>0$ such that for all $x, y \in \mathcal{H}$,

$$
u_{\infty}(x, y) \leq A\left(|x|^{2}+|y|^{2}+1\right) .
$$

(ii) For all $x, y \in \mathcal{H}$ we have

$$
u_{\infty}(x, y) \leq(|y|-1)^{2}-|x|^{2} .
$$

(iii) If $x, y, h, k \in \mathcal{H}$ satisfy

$$
|x|+|y| \leq 1, \quad|x+h|+|y+k| \geq 1 \quad \text { and } \quad|k| \leq|h|,
$$

then $u_{\infty}(x+h, y+k) \leq 0$.

(iv) If $x, y \in \mathcal{H}$ satisfy $|y| \leq|x|$, then $u_{\infty}(x, y) \leq 0$.

Proof (i), (ii) This follows immediately from the formula for $u_{\infty}$.

(iii) The desired inequality is equivalent to ||$y+k|-1| \leq|x+h|$. Note that $1-|y+k| \leq$ $|x+h|$, which is the middle bound in (2.5). In addition, combining this assumption with the triangle inequality, we obtain

$$
|y+k|-1 \leq|y|+|k|-1 \leq-|x|+|h| \leq|x+h|
$$

and we are done.

(iv) The estimate is trivial if $|x|+|y| \leq 1$; for remaining $x, y$,

$$
u_{\infty}(x, y)=(|y|+|x|-1)(|y|-|x|-1) \leq 0 .
$$

The key fact about $u_{\infty}$ is described in the following lemma.

Lemma 2.5 Suppose that martingales $X, Y$ are bounded in $L^{2}$ and $Y$ is differentially subordinate to $X$. Then for any $t \geq 0$,

$$
\mathbb{E} u_{\infty}\left(X_{t}, Y_{t}\right) \leq 0 .
$$

We shall need the following auxiliary fact. Recall that for any semimartingale $X$ there exists a unique continuous local martingale part $X^{c}$ of $X$ satisfying

$$
[X, X]_{t}=\left[X^{c}, X^{c}\right]_{t}+\sum_{0 \leq s \leq t}\left|\triangle X_{S}\right|^{2}
$$

for all $t \geq 0$. Here $\triangle X_{s}=X_{s}-X_{s-}$ denotes the jump of $X$ at time $s$ (we set $X_{0-} \equiv 0$ ). Furthermore, we have that $\left[X^{c}, X^{c}\right]=[X, X]^{c}$, the pathwise continuous part of $[X, X]$. Here is the Lemma 1 of Wang [17].

Lemma 2.6 If $X$ and $Y$ are semimartingales, then $Y$ is differentially subordinate to $X$ if and only if $Y^{c}$ is differentially subordinate to $X^{c}$ and $\left|\Delta Y_{s}\right| \leq\left|\Delta X_{s}\right|$ for all $s \geq 0$. 
Proof of Lemma 2.5 By (2.3), we see that the random variable $u_{\infty}\left(X_{t}, Y_{t}\right)$ is integrable. Introduce the stopping time $\tau=\inf \left\{s \geq 0:\left|X_{s}\right|+\left|Y_{s}\right|>1\right\}$. We shall show the following three statements:

$$
\begin{gathered}
\mathbb{E} u_{\infty}\left(X_{t}, Y_{t}\right) 1_{\left\{\left|X_{0}\right|+\left|Y_{0}\right|>1\right\}} \leq \mathbb{E} u_{\infty}\left(X_{0}, Y_{0}\right) 1_{\left\{\left|X_{0}\right|+\left|Y_{0}\right|>1\right\}}, \\
u_{\infty}\left(X_{t}, Y_{t}\right)=u_{\infty}\left(X_{0}, Y_{0}\right)=0 \quad \text { on }\left\{\left|X_{0}\right|+\left|Y_{0}\right| \leq 1, \tau>t\right\}
\end{gathered}
$$

and

$$
\mathbb{E} u_{\infty}\left(X_{t}, Y_{t}\right) 1_{\left\{\left|X_{0}\right|+\left|Y_{0}\right| \leq 1, \tau \leq t\right\}} \leq \mathbb{E} u_{\infty}\left(X_{0}, Y_{0}\right) 1_{\left\{\left|X_{0}\right|+\left|Y_{0}\right| \leq 1, \tau \leq t\right\}} .
$$

These three facts yield the claim: indeed, they give $\mathbb{E} u_{\infty}\left(X_{t}, Y_{t}\right) \leq \mathbb{E} u_{\infty}\left(X_{0}, Y_{0}\right)$ and it suffices to note that $u_{\infty}\left(X_{0}, Y_{0}\right) \leq 0$, in view of the differential subordination and part (iv) of Lemma 2.4.

To prove (2.7), use (2.4) to get

$$
\mathbb{E}\left[u_{\infty}\left(X_{t}, Y_{t}\right) \mid \mathcal{F}_{0}\right] \leq \mathbb{E}\left[\left|Y_{t}\right|^{2}-\left|X_{t}\right|^{2} \mid \mathcal{F}_{0}\right]-2 \mathbb{E}\left(\left|Y_{t}\right| \mid \mathcal{F}_{0}\right)+1 .
$$

Of course, $\mathbb{E}\left(\left|Y_{t}\right| \mid \mathcal{F}_{0}\right) \geq\left|Y_{0}\right|$. Moreover,

$$
\mathbb{E}\left[\left(\left|Y_{t}\right|^{2}-\left|X_{t}\right|^{2}\right)-\left(\left|Y_{0}\right|^{2}-\left|X_{0}\right|^{2}\right) \mid \mathcal{F}_{0}\right]=\mathbb{E}\left[\left([Y, Y]_{t}-[X, X]_{t}\right)-\left([Y, Y]_{0}-[X, X]_{0}\right) \mid \mathcal{F}_{0}\right]
$$

is nonpositive, due to the differential subordination. Consequently, on the set $\left\{\left|X_{0}\right|+\right.$ $\left.\left|Y_{0}\right|>1\right\}$,

$$
\mathbb{E}\left[u_{\infty}\left(X_{t}, Y_{t}\right) \mid \mathcal{F}_{0}\right] \leq\left|Y_{0}\right|^{2}-\left|X_{0}\right|^{2}-2\left|Y_{0}\right|+1=u_{\infty}\left(X_{0}, Y_{0}\right),
$$

which yields (2.7). The condition (2.8) is obvious, by the definition of $u_{\infty}$ and $\tau$. To get (2.9), we proceed as previously: on the set $\left\{\left|X_{0}\right|+\left|Y_{0}\right| \leq 1, \tau \leq t\right\}$ we have, in virtue of (2.4),

$$
\begin{aligned}
\mathbb{E}\left[u_{\infty}\left(X_{t}, Y_{t}\right) \mid \mathcal{F}_{\tau}\right] & =\mathbb{E}\left[\left|Y_{t}\right|^{2}-\left|X_{t}\right|^{2} \mid \mathcal{F}_{\tau}\right]-2 \mathbb{E}\left(\left|Y_{t}\right| \mid \mathcal{F}_{\tau}\right)+1 \\
& \leq\left|Y_{\tau}\right|^{2}-\left|X_{\tau}\right|^{2}-2\left|Y_{\tau}\right|+1 \\
& =u_{\infty}\left(X_{\tau}, Y_{\tau}\right) .
\end{aligned}
$$

Now use part (iii) of Lemma 2.4 with $x=X_{\tau-}, y=Y_{\tau-}, h=\Delta X_{\tau}$ and $k=\Delta Y_{\tau}$ : the first two conditions in (2.5) follow from the definition of $\tau$, while the third one, $\left|\Delta Y_{\tau}\right| \leq\left|\Delta X_{\tau}\right|$, is due to the differential subordination. Thus, $u_{\infty}\left(X_{\tau}, Y_{\tau}\right) \leq 0=u_{\infty}\left(X_{0}, Y_{0}\right)$ and the proof is complete.

Now fix $K>1$ and recall that $S=\{(x, y) \in \mathcal{H} \times \mathcal{H}:|x| \leq 1\}$. Introduce the function $U_{K}: S \rightarrow \mathbb{R}$ by

$$
U_{K}(x, y)=\int_{1}^{\infty} a_{K}(r) u_{\infty}(x / r, y / r) \mathrm{d} r+b_{K}\left(|y|^{2}-|x|^{2}\right)
$$

where

$$
a_{K}(r)=\frac{r^{2}}{2 K^{2}(K-1)} \exp \left(\frac{r}{K}-1\right) \quad \text { and } \quad b_{K}=\frac{1}{2 K(K-1)} .
$$

A direct computation shows that $U_{K}(x, y)=\left(|y|^{2}-|x|^{2}\right) /(2 K(K-1))$ if $|x|+|y| \leq 1$, while for remaining $(x, y) \in S$ we have 


$$
U_{K}(x, y)=\frac{K-|x|}{K-1} \exp \left(\frac{|x|+|y|-1}{K}\right)-1-\frac{y}{K}-\frac{1}{2 K(K-1)} .
$$

We shall establish the following majorization.

Lemma 2.7 For any $(x, y) \in S$ we have

$$
U_{K}(x, y) \geq \Phi(|y| / K)-|x| /(2 K(K-1)) .
$$

Proof Of course, it suffices to establish the inequality for $\mathcal{H}=\mathbb{R}$; furthermore, since $U_{K}(x, y)=U_{K}(-x, y)=U_{K}(x,-y)$ for all $(x, y) \in S$, we may restrict ourselves to $x, y \geq 0$. The next observation is that $U_{K}$ is linear along the line segments of slope -1 contained in the strip $S_{+}=[0,1] \times[0, \infty)$; thus it suffices to check the majorization at the boundary of $S_{+}$. Finally, we have that the function $x \mapsto U_{K}(x, 0)$ is concave on $[0,1]$ and hence we will be done if we show (2.10) for $x \in\{0,1\}$ and $y \geq 0$. We easily check that both sides of the estimate are equal when $x=1$. To deal with the case $x=0$, define $F(y)=U_{K}(0, y)-\Phi(y / K)$ for $y \geq 0$. One easily checks that $F$ is of class $C^{1}$, satisfies $F(0)=F^{\prime}(0+)=0$ and

$$
F^{\prime \prime}(y)= \begin{cases}K^{-2}\left(\frac{K}{K-1}-e^{y / K}\right) & \text { if } y<1 \\ K^{-2} e^{(y-1) / K}\left(\frac{K}{K-1}-e^{1 / K}\right) & \text { if } y>1\end{cases}
$$

Thus $F$ is convex and hence nonnegative. The claim follows.

Now we are ready to establish Theorem 2.3.

Proof of (2.2) Fix $X, Y$ as in the statement, $M>0$ and introduce the stopping time

$$
\tau=\tau_{M}=\inf \left\{t \geq 0:\left|Y_{t}\right| \geq M\right\} .
$$

Then the stopped martingale $Y^{\tau}=\left(Y_{\tau \wedge t}\right)_{t \geq 0}$ is bounded: indeed, for any $t$ we have $\left|Y_{\tau \wedge t-}\right| \leq M$, so by Lemma 2.6 ,

$$
\left|Y_{\tau \wedge t}\right| \leq\left|Y_{\tau \wedge t-}\right|+\left|\Delta Y_{\tau \wedge t}\right| \leq M+\left|\Delta X_{\tau \wedge t}\right| \leq M+2|| X \|_{\infty}
$$

Obviously, $Y^{\tau}$ is differentially subordinate to $X$. Thus $\left\|Y_{t}^{\tau}\right\|_{2} \leq\left\|X_{t}\right\|_{2}$, using the $L^{2}$ inequality of Burkholder. Hence, by Lemma 2.5 and Fubini's theorem,

$$
\mathbb{E} U_{K}\left(X_{t}, Y_{t}^{\tau}\right) \leq \int_{1}^{\infty} a_{K}(r) \mathbb{E} u_{\infty}\left(X_{t} / r, Y_{t}^{\tau} / r\right) \mathrm{d} r \leq 0 .
$$

The fact that Fubini's theorem is applicable follows immediately from the boundedness of $X$ and $Y^{\tau}$. Combining (2.11) with (2.10) yields

$$
\mathbb{E} \Phi\left(\left|Y_{t}^{\tau}\right| / K\right) \leq \frac{1}{2 K(K-1)} \mathbb{E}\left|X_{t}\right| \leq \frac{1}{2 K(K-1)}\|X\|_{1}
$$

It suffices to let $M \rightarrow \infty$ and then take supremum over all $t \geq 0$ to get the claim, in virtue of Fatou's lemma. 
Sharpness Fix $\delta>0$ and consider a sequence $\left(\xi_{n}\right)_{n \geq 0}$ of independent random variables given by $\xi_{0} \equiv 1 / 2, \mathbb{P}\left(\xi_{1}=-1 / 2\right)=\mathbb{P}\left(\xi_{1}=1 / 2\right)=1 / 2$ and, for $k \geq 1$,

$$
\begin{aligned}
\mathbb{P}\left(\xi_{2 k}=-1\right) & =1-\mathbb{P}\left(\xi_{2 k}=\delta\right)=\frac{\delta}{1+\delta}, \\
\mathbb{P}\left(\xi_{2 k+1}=1-\delta\right) & =1-\mathbb{P}\left(\xi_{2 k+1}=-\delta\right)=\delta .
\end{aligned}
$$

Introduce the stopping time $\tau=\inf \left\{k:(-1)^{k} \xi_{k}<0\right\}$, which, obviously, is finite almost surely. Define the processes $X, Y$ by

$$
X_{t}=\xi_{0}+\xi_{1}+\xi_{2}+\cdots+\xi_{\tau \wedge\lfloor t\rfloor}, \quad Y_{t}=\xi_{0}-\xi_{1}+\xi_{2}-\cdots+(-1)^{\tau \wedge\lfloor t\rfloor} \xi_{\tau \wedge\lfloor t\rfloor} .
$$

Let us gather some information about these processes. Since $\xi_{1}, \xi_{2}, \ldots$ have mean zero, $X$ and $Y$ are martingales. Furthermore, we have $[X, X]_{t}=[Y, Y]_{t}$ for all $t \geq 0$, so $Y$ is differentially subordinate to $X$. Next, it is not difficult to verify that $X$ is bounded by 1 and $Y_{\tau}$ takes values in the set $\{0,2 \delta, 4 \delta, \ldots\}$. More precisely,

$$
\mathbb{P}\left(Y_{\tau}=0\right)=\mathbb{P}(\tau=1)+\mathbb{P}(\tau=2)=\frac{1}{2}+\frac{1}{2} \cdot \frac{\delta}{1+\delta}
$$

and, for $\ell=1,2, \ldots$,

$$
\mathbb{P}\left(Y_{\tau}=2 \ell \delta\right)=\mathbb{P}(\tau=2 \ell+1)+\mathbb{P}(\tau=2 \ell+2)=\frac{\delta(1-\delta)^{\ell-1}}{(1+\delta)^{\ell+1}}
$$

Consequently, for $K>1$ we have

$$
\mathbb{E} \Phi\left(Y_{\tau} / K\right)=\frac{\delta}{1-\delta^{2}} \sum_{\ell \geq 1} \Phi(2 \ell \delta / K)\left(1-\frac{2 \delta}{1+\delta}\right)^{\ell}
$$

If we let $\delta \rightarrow 0$, the expression on the right converges to

$$
\frac{1}{2} \int_{0}^{\infty} \Phi(x / K) e^{-x} \mathrm{~d} x=\frac{1}{2 K(K-1)},
$$

and thus the constant $(2 K(K-1))^{-1}$ cannot be improved in (2.2).

\section{Proof of Theorem 1.2}

We start by recalling the martingale representation of the multipliers from the class (1.1). This is described in full detail in [1] and [2], so we shall be brief. Let $m$ be the multiplier as in (1.1), with the corresponding parameters $\phi, \psi, \mu$ and $\nu$. Assume in addition that $v\left(\mathbb{R}^{d}\right)$ is finite and nonzero. Then for any $s<0$ there is a Lévy process $\left(X_{s, t}\right)_{t \in[s, 0]}$ with $X_{s, s} \equiv 0$, for which Lemmas 3.1 and 3.2 below hold true. To state these, we need some notation. For a given $f \in L^{\infty}\left(\mathbb{R}^{d}\right)$, define the corresponding parabolic extension $\mathcal{U}_{f}$ to $(-\infty, 0] \times \mathbb{R}^{d}$ by

$$
\mathcal{U}_{f}(s, x)=\mathbb{E} f\left(x+X_{s, 0}\right) .
$$


Next, fix $x \in \mathbb{R}^{d}, s<0$ and let $f, \phi \in L^{\infty}\left(\mathbb{R}^{d}\right)$. We introduce the processes $F=$ $\left(F_{t}^{x, s, f}\right)_{s \leq t \leq 0}$ and $G=\left(G_{t}^{x, s, f, \phi}\right)_{s \leq t \leq 0}$ by

$$
\begin{aligned}
F_{t}= & \mathcal{U}_{f}\left(t, x+X_{s, t}\right), \\
G_{t}= & \sum_{s<u \leq t}\left[\left(F_{u}-F_{u-}\right) \cdot \phi\left(X_{s, u}-X_{s, u-}\right)\right] \\
& \quad-\int_{s}^{t} \int_{\mathbb{R}^{d}}\left[\mathcal{U}_{f}\left(v, x+X_{s, v-}+z\right)-\mathcal{U}_{f}\left(v, x+X_{s, v-}\right)\right] \phi(z) v(\mathrm{~d} z) \mathrm{d} v .
\end{aligned}
$$

Finally, fix $s<0$, a function $\phi$ on $\mathbb{R}^{d}$ taking values in the unit ball of $\mathbb{C}$ and define the operator $\mathcal{T}=\mathcal{T}^{s}$ by the bilinear form

$$
\int_{\mathbb{R}^{d}} \mathcal{T} f(x) g(x) \mathrm{d} x=\int_{\mathbb{R}^{d}} \mathbb{E}\left[G_{0}^{x, s, f, \phi} g\left(x+X_{s, 0}\right)\right] \mathrm{d} x,
$$

where $f, g \in C_{0}^{\infty}\left(\mathbb{R}^{d}\right)$. By the results from [1] and [2], the family $\left\{\left(X_{s, t}\right)_{s \leq t \leq 0}\right\}_{s<0}$ can be chosen so that the following statements are valid.

Lemma 3.1 For any fixed $x, s, f, \phi$ as above, the processes $F^{x, s, f}, G^{x, s, f, \phi}$ are martingales with respect to $\left(\mathcal{F}_{t}\right)_{s \leq t \leq 0}=\left(\sigma\left(X_{s, t}: s \leq t\right)\right)_{s \leq t \leq 0}$. Furthermore, if $\|\phi\|_{\infty} \leq 1$, then $G^{x, s, f, \phi}$ is differentially subordinate to $F$.

Lemma 3.2 Let $1<p<\infty$ and $d \geq 2$. The operator $\mathcal{T}^{s}$ is well defined and extends to a bounded operator on $L^{p}\left(\mathbb{R}^{d}\right)$, which can be expressed as a Fourier multiplier with the symbol

$$
\begin{aligned}
M(\xi) & =M_{s}(\xi) \\
& =\left[1-\exp \left(2 s \int_{\mathbb{R}^{d}}(1-\cos \langle\xi, z\rangle) v(d z)\right)\right] \frac{\int_{\mathbb{R}^{d}}(1-\cos \langle\xi, z\rangle) \phi(z) v(d z)}{\int_{\mathbb{R}^{d}}(1-\cos \langle\xi, z\rangle) v(d z)}
\end{aligned}
$$

if $\int_{\mathbb{R}^{d}}(1-\cos \langle\xi, z\rangle) v(d z) \neq 0$, and $M(\xi)=0$ otherwise.

We are ready to establish the following dual version of (1.3).

Theorem 3.3 Assume that $K>1$ and let $m: \mathbb{R}^{d} \rightarrow \mathbb{C}$ be a multiplier as in Theorem 1.2. Then for any Borel function $f$ on $\mathbb{R}^{d}$ taking values in the unit ball of $\mathbb{C}$ we have

$$
\left\|\Phi\left(\left|T_{m} f\right| / K\right)\right\|_{L^{1}\left(\mathbb{R}^{d}\right)} \leq \frac{1}{2 K(K-1)}\|f\|_{L^{1}\left(\mathbb{R}^{d}\right)} .
$$

Proof It is convenient to split the reasoning into two parts.

Step 1. First we show the estimate for the multipliers of the form

$$
M_{\phi, v}(\xi)=\frac{\int_{\mathbb{R}^{d}}(1-\cos \langle\xi, z\rangle) \phi(z) v(\mathrm{~d} z)}{\int_{\mathbb{R}^{d}}(1-\cos \langle\xi, z\rangle) v(\mathrm{~d} z)} .
$$

In addition, we assume that $0<v\left(\mathbb{R}^{d}\right)<\infty$, so that the above approach using Lévy processes is applicable. Fix $s<0$ and functions $f, g \in C_{0}^{\infty}\left(\mathbb{R}^{d}\right)$ such that $f$ is bounded 
by 1 ; then the martingale $F^{x, s, f}$ also takes values in the unit ball of $\mathbb{C}$.By Young inequality,

Fubini's theorem and (2.2), we have

$$
\begin{aligned}
& \left|\int_{\mathbb{R}^{d}} \mathbb{E}\left[\frac{G_{0}^{x, s, f, \phi}}{K} g\left(x+X_{s, 0}\right)\right] \mathrm{d} x\right| \\
& \quad \leq \int_{\mathbb{R}^{d}} \mathbb{E} \Phi\left(\frac{\left|G_{0}^{x, s, f, \phi}\right|}{K}\right) \mathrm{d} x+\int_{\mathbb{R}^{d}} \mathbb{E} \Psi\left(\left|g\left(x+X_{s, 0}\right)\right|\right) \mathrm{d} x \\
& \leq \frac{1}{2 K(K-1)} \int_{\mathbb{R}^{d}} \mathbb{E}\left|F_{0}^{x, s, f}\right| \mathrm{d} x+\int_{\mathbb{R}^{d}} \Psi(|g(x)|) \mathrm{d} x \\
& =\frac{1}{2 K(K-1)} \int_{\mathbb{R}^{d}}|f(x)| \mathrm{d} x+\int_{\mathbb{R}^{d}} \Psi(|g(x)|) \mathrm{d} x .
\end{aligned}
$$

Plugging this into the definition of $\mathcal{T}^{s}[($ see (3.2)], we obtain

$$
\int_{\mathbb{R}^{d}}\left[\frac{\mathcal{T}^{s} f(x)}{K} g(x)-\Psi(|g(x)|)\right] \mathrm{d} x \leq \frac{\|f\|_{L^{1}\left(\mathbb{R}^{d}\right)}}{2 K(K-1)} .
$$

Now fix $M>0$ and put

$$
g(x)=\frac{\overline{\mathcal{T}^{s} f(x)}}{\left|\mathcal{T}^{s} f(x)\right|}\left[\exp \left(\min \left\{\frac{\left|\mathcal{T}^{s} f(x)\right|}{K}, M\right\}\right)-1\right]
$$

(if $\mathcal{T}^{s} f(x)=0$, set $g(x)=0$ ). This gives

$$
\begin{aligned}
& \int_{\mathbb{R}^{d}} \Phi\left(\left|\frac{\mathcal{T}^{s} f(x)}{K}\right|\right) 1_{\left\{\left|\mathcal{T}^{s} f(x)\right| \leq M K\right\}} \\
& \quad+\left(\frac{\left|\mathcal{T}^{s} f(x)\right|\left(e^{M}-1\right)}{K}-\Psi\left(e^{M}-1\right)\right) 1_{\left\{\left|\mathcal{T}^{s} f(x)\right|>M K\right\}} \mathrm{d} x \leq \frac{\|f\|_{L^{1}\left(\mathbb{R}^{d}\right)}}{2 K(K-1)}
\end{aligned}
$$

and hence, by Fatou's lemma, if we let $M \rightarrow \infty$, we get

$$
\int_{\mathbb{R}^{d}} \Phi\left(\left|\frac{\mathcal{T}^{s} f(x)}{K}\right|\right) \mathrm{d} x \leq \frac{\|f\|_{L^{1}\left(\mathbb{R}^{d}\right)}}{2 K(K-1)} .
$$

Now if we let $s \rightarrow-\infty$,then $M_{s}$ converges pointwise to the multiplier $M_{\phi, v}$ given by (3.4). By Plancherel's theorem, $\mathcal{T}^{s} f \rightarrow T_{M_{\phi, v}} f$ in $L^{2}$ and hence there is a sequence $\left(s_{n}\right)_{n=1}^{\infty}$ converging to $-\infty$ such that $\lim _{n \rightarrow \infty} \mathcal{T}^{s_{n}} f \rightarrow T_{M_{\phi, v}} f$ almost everywhere. Thus Fatou's lemma yields the desired bound for the multiplier $T_{M_{\phi, v}}$.

Step 2. Now we deduce the result for the general multipliers as in (1.1) and drop the assumption $0<v\left(\mathbb{R}^{d}\right)<\infty$. For a given $\varepsilon>0$, define a Lévy measure $v_{\varepsilon}$ in polar coordinates $(r, \theta) \in(0, \infty) \times \mathbb{S}$ by

$$
v_{\varepsilon}(\mathrm{d} r \mathrm{~d} \theta)=\varepsilon^{-2} \delta_{\varepsilon}(\mathrm{d} r) \mu(d \theta),
$$


where $\delta_{\varepsilon}$ denotes Dirac measure on $\{\varepsilon\}$. Next, consider a multiplier $m_{\varepsilon}$ as in (3.4), in which the Lévy measure is $1_{\{|x|>\varepsilon\}} v+v_{\varepsilon}$ and the jump modulator is given by $1_{\{|x|>\varepsilon\}} \phi(x)+$ $1_{\{|x|=\varepsilon\}} \psi(x /|x|)$. If we let $\varepsilon \rightarrow 0$, we see that

$$
\begin{aligned}
\int_{\mathbb{R}^{d}}[1-\cos \langle\xi, x\rangle] \psi(x /|x|) v_{\varepsilon}(\mathrm{d} x)= & \int_{\mathbb{S}}\langle\xi, \theta\rangle^{2} \phi(\theta) \frac{1-\cos \langle\xi, \varepsilon \theta\rangle}{\langle\xi, \varepsilon \theta\rangle^{2}} \mu(d \theta) \\
& \rightarrow \frac{1}{2} \int_{\mathbb{S}}\langle\xi, \theta\rangle^{2} \phi(\theta) \mu(\mathrm{d} \theta) .
\end{aligned}
$$

This yields the claim by the similar argument as above, using of Plancherel's theorem and the passage to the subsequence which converges almost everywhere.

Proof of Theorem 1.2. For notational simplicity, we shall skip the lower indices and write $m$ instead of $m_{\phi, \psi, \mu, \nu}$. Observe that the class (1.1) is closed under the complex conjugation: we have $\bar{m}=m_{\bar{\phi}, \bar{\psi}, \mu, v}$. Fix $f \in L \log L\left(\mathbb{R}^{d}\right)$ and put $g=T_{m} f 1_{A} /\left|T_{m} f\right|(g=0$ if the denominator is zero). We have

$$
\begin{aligned}
\int_{A}\left|T_{m} f(x)\right| \mathrm{d} x & =\int_{\mathbb{R}^{d}} T_{m} f(x) \overline{g(x)} \mathrm{d} x \\
& =\int_{\mathbb{R}^{d}} \widehat{T_{m} f}(x) \overline{\widehat{g}(x)} \mathrm{d} x \\
& =\int_{\mathbb{R}^{d}} \widehat{f}(x) \overline{\widehat{T_{\bar{m}}}(x)} \mathrm{d} x \\
& =\int_{\mathbb{R}^{d}} f(x) \overline{T_{\bar{m}} g(x)} \mathrm{d} x \\
& \leq K \int_{\mathbb{R}^{d}} \Psi(|f(x)|) \mathrm{d} x+K \int_{\mathbb{R}^{d}} \Phi\left(\left|T_{\bar{m}} g(x)\right| / K\right) \mathrm{d} x \\
& \leq K \int_{\mathbb{R}^{d}} \Psi(|f(x)|) \mathrm{d} x+\frac{\|\|_{L^{1}\left(\mathbb{R}^{d}\right)}}{2(K-1)} .
\end{aligned}
$$

Here in the fifth line we have exploited Young's inequality and in the latter passage we have used (3.3) and the fact that $g$ takes values in the unit ball of $\mathbb{C}$. It suffices to note that $\|g\|_{L^{1}\left(\mathbb{R}^{d}\right)} \leq|A|$ to complete the proof.

In the remainder of this section we discuss the possibility of extending the assertion of Theorem 1.2 to the vector-valued multipliers. For any bounded function $m=\left(m_{1}, m_{2}, \ldots, m_{n}\right)$ : $\mathbb{R}^{d} \rightarrow \mathbb{C}^{n}$, we may define the associated Fourier multiplier acting on complex valued functions on $\mathbb{R}^{d}$ by the formula $T_{m} f=\left(T_{m_{1}} f, T_{m_{2}} f, \ldots, T_{m_{n}} f\right)$. As we shall see, the reasoning presented above can be easily modified to yield the following statement.

Theorem 3.4 Let $v, \mu$ be two measures on $\mathbb{R}^{d}$ and $\mathbb{S}$, respectively, satisfying the assumptions of Theorem 1.2. Assume further that $\phi, \psi$ are two Borel functions on $\mathbb{R}^{d}$ taking values in the unit ball of $\mathbb{C}^{n}$ and let $m: \mathbb{R}^{d} \rightarrow \mathbb{C}^{n}$ be the associated symbol given by (1.1). Then for any complex valued function $f$ on $\mathbb{R}^{d}$ and $K>1$ we have 


$$
\int_{\mathbb{R}^{d}} \exp \left(\left|\frac{T_{m} f(x)}{K}\right|\right) d x \leq \frac{\|f\|_{L^{1}\left(\mathbb{R}^{d}\right)}}{2 K(K-1)}
$$

and for any Borel subset $A$ of $\mathbb{R}^{d}$,

$$
\int_{A}\left|T_{m} f(x)\right| d x \leq K \int_{\mathbb{R}^{d}} \Psi(|f(x)|) d x+\frac{|A|}{2(K-1)} .
$$

Proof Suppose first that $v$ is finite. For a given $C^{\infty}$ function $f: \mathbb{R}^{d} \rightarrow \mathbb{C}$ bounded by 1 , we introduce martingales $F$ and $G=\left(G^{1}, G^{2}, \ldots, G^{n}\right)$ by the formula (3.1). It is easy to check that $G$ is differentially subordinate to $F$, arguing as in [1] or [2]. Applying the representation (3.2) to each coordinate of $G$ separately, we obtain the associated multiplier $\mathcal{T}=\left(\mathcal{T}^{1}, \mathcal{T}^{2}, \ldots, \mathcal{T}^{n}\right)$, where $\mathcal{T}^{j}$ has symbol $M_{\phi_{j}, v_{j}}$ defined in (3.4). Now we repeat the reasoning from (3.5), with a vector valued function $g: \mathbb{R}^{d} \rightarrow \mathbb{C}^{n}$ (the expression $G_{0}^{x, s, f, \phi} g\left(x+X_{s, 0}\right)$ under the first integral is replaced with the corresponding scalar product). An application of (2.2) gives

$$
\|\Phi(|\mathcal{T} f| / K)\|_{L^{1}\left(\mathbb{R}^{d} ; \mathbb{C}^{n}\right)} \leq \frac{\|f\|_{L^{1}\left(\mathbb{R}^{d}\right)}}{2 K(K-1)},
$$

which extends to general $f$ by standard density arguments. The passage to general $m$ as in (1.1) is carried over in the same manner as in the scalar case and thus we obtain the vector version of Theorem 3.3. The duality argument explained in (3.6) extends to the vectorvalued setting with no difficulties (one only has to replace appropriate multiplications by scalar products) and thus Theorem 1.2 holds true for the multipliers in $\mathbb{C}^{n}$.

\section{Logarithmic estimates for the Beurling-Ahlfors operator}

The inequalities obtained in the previous sections can be applied to the Beurling-Ahlfors transform $\mathcal{B A}$ on $\mathbb{C}$. This operator is a Fourier multiplier with the symbol $m(\xi)=\bar{\xi} / \xi, \xi \in \mathbb{C}$; alternatively, it can be defined by the singular integral

$$
\mathcal{B} \mathcal{A} f(z)=-\frac{1}{\pi} \text { p.v. } \int_{\mathbb{C}} \frac{f(w)}{(z-w)^{2}} \mathrm{~d} w .
$$

The Beurling-Ahlfors transform is of fundamental importance in the study of partial differential equations and quasiconformal mappings, since it changes the complex derivative $\bar{\partial}$ to д. Precisely, we have

$$
\mathcal{B A}(\bar{\partial} f)=\partial f
$$

for any $f$ from the Sobolev space $W^{1,2}(\mathbb{C}, \mathbb{C})$ of complex valued locally integrable functions on $\mathbb{C}$ whose distributional first derivatives are in $L^{2}$ on the plane. Directly from the form of the multiplier $m$ we infer that $\mathcal{B A}$ is an isometry on $L^{2}(\mathbb{C})$ and $\|\mathcal{B A}\|_{L^{2}(\mathbb{C}) \rightarrow L^{2}(\mathbb{C})}=1$. There is a very interesting question about the norm of the Beurling-Ahlfors operator acting on $L^{p}(\mathbb{C}), 1<p<\infty$. A long-standing conjecture of T. Iwaniec [11] states that $\|\mathcal{B A}\|_{L^{p}(\mathbb{C}) \rightarrow L^{p}(\mathbb{C})}=p^{*}-1$ for all $p$ from that range. In 1965, Lehto constructed some examples showing that $\|\mathcal{B} \mathcal{A}\|_{L^{p}(\mathbb{C}) \rightarrow L^{p}(\mathbb{C})} \geq p^{*}-1$. The upper bound has been studied by many authors: see e.g. $[3,6,10]$ and [13]. The most tight result so far is the estimate 
$\|\mathcal{B A}\|_{L^{p}(\mathbb{C}) \rightarrow L^{p}(\mathbb{C})} \leq 1.575\left(p^{*}-1\right)$ of Bañuelos and Janakiraman, who established it by a clever refinement of Burkholder's moment $L^{p}$ estimate.

The results of this paper yield localized logarithmic estimates for the Beurling-Ahlfors operator. Write the identity

$$
\frac{\bar{\xi}}{\xi}=\frac{\xi_{1}^{2}-\xi_{2}^{2}}{\xi_{1}^{2}+\xi_{2}^{2}}+i \frac{2 \xi_{1} \xi_{2}}{\xi_{1}^{2}+\xi_{2}^{2}}
$$

and note that the real and imaginary parts of $\bar{\xi} / \xi$ can be represented as the Fourier multipliers with the symbols of the form (1.1). For example, the choice $d=2, \mu=$ $\delta_{(1,0)}+\delta_{(0,1)}, \psi(1,0)=-1=-\psi(0,1)$ and $v=0$ gives rise to $T_{m}=\operatorname{Re}(\mathcal{B A})$; likewise, $d=2, \mu=\delta_{(1 / \sqrt{2}, 1 / \sqrt{2})}+\delta_{(1 / \sqrt{2},-1 / \sqrt{2})}, \psi(1 / \sqrt{2}, 1 / \sqrt{2})=1-\psi(1 / \sqrt{2},-1 / \sqrt{2})$ and $v=0$ leads to $T_{m}=\operatorname{Im}(\mathcal{B A})$. By a similar choice of the parameters, $\frac{1}{2} \mathcal{B A}$ can also be shown to be a multiplier of the form described in (1.1).

Thus, the following statement is an immediate consequence of Theorem 1.2.

Theorem 4.1 For any $K>1$ and any Borel subset $A$ of $\mathbb{C}$ we have

$$
\begin{aligned}
& \int_{A}|\operatorname{Re}(\mathcal{B A}) f(z)| d z \leq K \int_{\mathbb{C}} \Psi(|f(z)|) d z+\frac{|A|}{2(K-1)}, \\
& \int_{A}|\operatorname{Im}(\mathcal{B A}) f(z)| d z \leq K \int_{\mathbb{C}} \Psi(|f(z)|) d z+\frac{|A|}{2(K-1)}
\end{aligned}
$$

and

$$
\int_{A}|\mathcal{B} \mathcal{A} f(z)| d z \leq 2 K \int_{\mathbb{C}} \Psi(|f(z)|) d z+\frac{|A|}{K-1} .
$$

We turn to the lower bounds. The following statement immediately yields the assertion of Theorem 1.4.

Theorem 4.2 (i) For any $K>1$ there is a function $f \in W^{1,2}(\mathbb{C}, \mathbb{C})$ and a Borel subset A of $\mathbb{C}$ such that

$$
\int_{A}|\mathcal{B A} f(z)| d z=K \int_{\mathbb{C}} \Psi(|f(z)|) d z+\frac{|A|}{4(K-1)} .
$$

(ii) For any $K>2 / \pi$ there is a function $f \in W^{1,2}(\mathbb{C}, \mathbb{C})$ and a Borel subset $A$ of $\mathbb{C}$ such that

$$
\begin{aligned}
& \int_{A}|\operatorname{Re}(\mathcal{B A}) f(z)| d z=K \int_{\mathbb{C}} \Psi(|f(z)|) d z+\frac{|A|}{\pi(K \pi-2)}, \\
& \int_{A}|\operatorname{Im}(\mathcal{B A}) f(z)| d z=K \int_{\mathbb{C}} \Psi(|f(z)|) d z+\frac{|A|}{\pi(K \pi-2)} .
\end{aligned}
$$


(iii) For any $c<2 / \pi$ there is a function $f \in W^{1,2}(\mathbb{C}, \mathbb{C})$ and a Borel subset $A$ of $\mathbb{C}$ such that

$$
\begin{aligned}
& \int_{A}|\operatorname{Re}(\mathcal{B} \mathcal{A}) f(z)| d z>\int_{\mathbb{C}} \Psi(|f(z)|) d z+c\left(|A| \int_{\mathbb{C}} \Psi(|f(z)|) d z\right), \\
& \int_{A}|\operatorname{Im}(\mathcal{B} \mathcal{A}) f(z)| d z>\int_{\mathbb{C}} \Psi(|f(z)|) d z+c\left(|A| \int_{\mathbb{C}} \Psi(|f(z)|) d z\right) .
\end{aligned}
$$

Proof For a fixed $\alpha \in(0,1)$, let $R>0$ be given by the equation $R^{2 \alpha}=1-\alpha$. Consider a function $w: \mathbb{C} \rightarrow \mathbb{C}$ defined by

$$
w(z)= \begin{cases}\bar{z}|z|^{-2 \alpha}-\bar{z} & \text { if }|z| \leq R \\ R^{2-2 \alpha} z^{-1}-R^{2} z^{-1} & \text { if }|z|>R\end{cases}
$$

We easily check that $w \in W^{1,2}(\mathbb{C}, \mathbb{C})$ and derive that

$$
\partial w(z)= \begin{cases}\alpha \bar{z}^{2}|z|^{-2 \alpha-2} & \text { if }|z|<R \\ -R^{2-2 \alpha} z^{-2}+R^{2} z^{-2} & \text { if }|z|>R\end{cases}
$$

and

$$
\bar{\partial} w(z)= \begin{cases}(1-\alpha)|z|^{-2 \alpha}-1 & \text { if }|z|<R, \\ 0 & \text { if }|z|>R .\end{cases}
$$

Finally, put $A=\{z \in \mathbb{C}:|z| \leq R\}$ and $f=\bar{\partial} w$. We derive that

$$
\int_{\mathbb{C}} \Psi(|f(z)|) \mathrm{d} z=\pi R^{2-2 \alpha}\left[\log (1-\alpha)+\frac{2 \alpha-1}{1-\alpha}-2 \alpha \log R\right]+\pi R^{2}=\frac{\alpha^{2}|A|}{(1-\alpha)^{2}} .
$$

Now we consider the parts (i), (ii) and (iii) separately.

(i) Let $\alpha=(2 K-1)^{-1}$. Using the polar coordinates, we easily compute that

$$
\int_{A}|\mathcal{B A} f(z)| \mathrm{d} z=\int_{A}|\partial w(z)| \mathrm{d} z=\frac{\pi \alpha}{1-\alpha} R^{2-2 \alpha}=|A| \cdot \frac{2 K-1}{4(K-1)^{2}}
$$

and

$$
\int_{\mathbb{C}} \Psi(|f(z)|) \mathrm{d} z=|A| \cdot \frac{1}{4(K-1)^{2}} .
$$

Now (4.2) can be verified readily.

(ii) Let $\alpha=(K \pi-1)^{-1}$. It is straightforward to derive that

$$
\int_{A}|\operatorname{Re}(\mathcal{B} \mathcal{A}) f(z)| \mathrm{d} z=\frac{2 \alpha}{1-\alpha} R^{2-2 \alpha}=|A| \cdot \frac{2(K \pi-1)}{\pi(K \pi-2)^{2}}
$$

and

$$
\int_{\mathbb{C}} \Psi(|f(z)|) \mathrm{d} z=|A| \cdot \frac{1}{(K \pi-2)^{2}}
$$


This gives the first equality in (4.3). The second one is established analogously, with the same choice of $\alpha$.

(iii) We compute that

$$
\frac{\int_{A}|\operatorname{Re}(\mathcal{B A}) f(z)| \mathrm{d} z-\int_{\mathbb{C}} \Psi(|f(z)|) \mathrm{d} z}{\left(|A| \int_{\mathbb{C}} \Psi(|f(z)|) \mathrm{d} z\right)^{1 / 2}}=\frac{2-\pi \alpha}{\pi(1-\alpha)},
$$

which can be made arbitrarily close to $2 / \pi$ by choosing a sufficiently small $\alpha$. The reasoning for the second bound in (4.4) is similar. This completes the proof of the theorem.

Finally, let us address the questions raised below the statement of Theorem 1.2. Since $\frac{1}{2} \mathcal{B A}$ belongs to the class of multipliers with the symbols of the form (1.1), it suffices to establish the following fact.

Theorem 4.3 (i) For any $c>0$ and $K>0$ there is a Borel subset $A$ of $\mathbb{C}$ and a Borel function $f$ on $\mathbb{C}$ for which

$$
\int_{A}|\mathcal{B A} f(z)| d z>K \int_{\mathbb{C}}|f(z)| \log ^{+}|f(z)| d z+c|A| .
$$

(ii) For any $c>0$ and $K>0$ there is a Borel subset $A$ of $\mathbb{C}$ and a Borel function $f$ on $\mathbb{C}$ for which

$$
\int_{A}|\mathcal{B} \mathcal{A} f(z)| d z>K \int_{A} \Psi(|f(z)|) d z+c|A| .
$$

Proof Introduce $w \in W^{1,2}(\mathbb{C}, \mathbb{C})$ by $w(z)=\left(z \log |z|^{2}\right) 1_{\{|z| \leq 1\}}$ and let $f=\bar{\partial} w$. We easily check that

$$
f(z)=(z /|z|)^{2} 1_{\{|z| \leq 1\}} \quad \text { and } \quad \mathcal{B A} f(z)=\partial w(z)=\left(1+\log |z|^{2}\right) 1_{\{|z| \leq 1\}} .
$$

In particular, $|f| \leq 1$ on $\mathbb{C}$ and hence we will be done if we show that the expression

$$
\frac{1}{|A|} \int_{A}|\mathcal{B A} f(z)| \mathrm{d} z
$$

can be made arbitrarily large by the appropriate choice of a set $A$. But this is evident: since $\lim _{z \rightarrow 0}|\mathcal{B A} f(z)|=\infty$, it suffices to take $A=\{z \in \mathbb{C}:|z| \leq r\}$ for $r>0$ small enough.

Acknowledgments The author would like to thank the anonymous Referee for the careful reading of the first version of the paper and some helpful suggestions.

Open Access This article is distributed under the terms of the Creative Commons Attribution License which permits any use, distribution, and reproduction in any medium, provided the original author(s) and the source are credited.

\section{References}

1. Bañuelos, R., Bogdan, K.: Lévy processes and Fourier multipliers. J. Funct. Anal. 250, 197-213 (2007)

2. Bañuelos, R., Bielaszewski, A., Bogdan, K.: Fourier multipliers for non-symmetric Lévy processes. Marcinkiewicz centenary volume, pp 9-25, Banach Center Publications, 95, Warsaw (2011) 
3. Bañuelos, R., Méndez-Hernández, P.: Space-time Brownian motion and the Beurling-Ahlfors transform. Indiana Univ. Math. J. 52, 981-990 (2003)

4. Bañuelos, R., Janakiraman, P.: $L_{p}$-bounds for the Beurling-Ahlfors transform. Trans. Amer. Math. Soc. 360, 3603-3612 (2008)

5. Bañuelos, R., Osȩkowski, R.: Martingales and sharp bounds for Fourier multipliers. to appear in Ann. Acad. Sci. Fenn. Math.

6. Bañuelos, R., Wang, G.: Sharp inequalities for martingales with applications to the Beurling-Ahlfors and Riesz transformations. Duke Math. J 80, 575-600 (1995)

7. Burkholder, D.L.: Boundary value problems and sharp inequalities for martingale transform. Ann. Probab. 12, 647-702 (1984)

8. Burkholder, D.L.: A sharp and strict $L^{p}$-inequality for stochastic integrals. Ann. Probab. 15, 268-273 (1987)

9. Dellacherie, C., Meyer, P.-A.: Probabilities and potential B: theory of martingales. North Holland, Amsterdam (1982)

10. Geiss, E., Mongomery-Smith, S., Saksman, E.: On singular integral and martingale transforms. Trans. Amer. Math. Soc. 362, 553-575 (2010)

11. Iwaniec, T.: Extremal inequalities in Sobolev spaces and quasiconformal mappings. Z. Anal. Anwendungen 1, 1-16 (1982)

12. Lehto, O.: Remarks on the integrability of the derivatives of quasiconformal mappings. Ann. Acad. Sci. Fenn. Series AI Math. 371, 3-8 (1965)

13. Nazarov, F., Volberg, A.: Heat extension of the Beurling operator and estimates for its norm. St. Petersburg Math. J. 15, 563-573 (2004)

14. Osȩkowski, A.: Sharp LlogL inequality for Differentially Subordinated Martingales. Ill J. Math., 52 3, 745-756, (2008)

15. Osȩkowski, A.: On relaxing the assumption of differential subordination in some martingale inequalities. Electr. Commun. Probab. 15, 9-21, (2011)

16. Osȩkowski, A.: Logarithmic estimates for submartingales and their differential subordinates. J. Theor. Probab. 24, 849-874, (2011)

17. Wang, G.: Differential subordination and strong differential subordination for continuous time martingales and related sharp inequalities. Ann. Probab. 23, 522-551 (1995) 